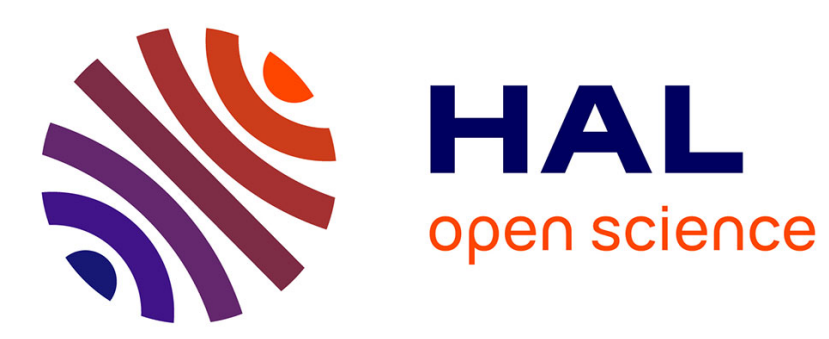

\title{
On the mortality problem for matrices of low dimensions
} Olivier Bournez, Michael Branicky

\section{To cite this version:}

Olivier Bournez, Michael Branicky. On the mortality problem for matrices of low dimensions. [Research Report] LIP RR-1999-20, Laboratoire de l'informatique du parallélisme. 1999, 2+13p. hal02101904

\section{HAL Id: hal-02101904 \\ https://hal-lara.archives-ouvertes.fr/hal-02101904}

Submitted on 17 Apr 2019

HAL is a multi-disciplinary open access archive for the deposit and dissemination of scientific research documents, whether they are published or not. The documents may come from teaching and research institutions in France or abroad, or from public or private research centers.
L'archive ouverte pluridisciplinaire HAL, est destinée au dépôt et à la diffusion de documents scientifiques de niveau recherche, publiés ou non, émanant des établissements d'enseignement et de recherche français ou étrangers, des laboratoires publics ou privés. 
Laboratoire de l'Informatique du Parallélisme

École Normale Supérieure de Lyon

Unité Mixte de Recherche CNRS-INRIA-ENS LYON

$\mathrm{n}^{\mathrm{o}} 5668$

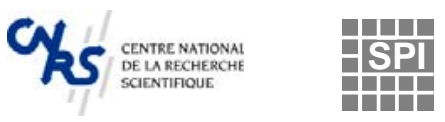

On the mortality problem for matrices of low dimensions

Olivier Bournez

Michael Branicky

January 1999

École Normale Supérieure de Lyon

46 Allée d'Italie, 69364 Lyon Cedex 07, France

Téléphone : +33(0)4.72.72.80.37

Télécopieur : $+33(0) 4.72 .72 .80 .80$

Adresse électronique : lip@ens-lyon.fr 


\title{
On the mortality problem for matrices of low dimensions
}

\author{
Olivier Bournez \\ Michael Branicky
}

January 1999

\begin{abstract}
In this paper, we discuss the existence of an algorithm to decide if a given set of $2 \times 2$ matrices is mortal: a set $F=\left\{A_{1}, \ldots, A_{m}\right\}$ of $2 \times 2$ matrices is said to be mortal if there exist an integer $k \geq 1$ and some integers $i_{1}, i_{2}, \ldots, i_{k} \in$ $\{1, \ldots, m\}$ with $A_{i_{1}} A_{i_{2}} \cdots A_{i_{k}}=0$. We survey this problem and propose some new extensions: we prove the problem to be BSS-undecidable for real matrices and Turing-decidable for two rational matrices. We relate the problem for rational matrices to the entry equivalence problem, to the zero in the left upper corner problem and to the reachability problem for piecewise affine functions. Finally, we state some NP-completeness results.
\end{abstract}

Keywords: Mortality, Matrices, Decidability

\section{Résumé}

Dans ce papier, nous discutons l'existence d'un algorithme pour décider si un ensemble donné de matrices $2 \times 2$ est mortel: un ensemble $F=\left\{A_{1}, \ldots, A_{m}\right\}$ de matrices $2 \times 2$ est dit mortel s'il existe un entier $k \geq 1$ et des entiers $i_{1}, i_{2}, \ldots, i_{k} \in\{1, \ldots, m\}$ avec $A_{i_{1}} A_{i_{2}} \cdots A_{i_{k}}=0$. Nous présentons une synthèse des résultats connus sur ce problème et présentons quelques extensions: nous prouvons que le problème est BSS-indécidable pour les matrices réelles et Turing-décidable pour les matrices rationnelles. Nous relions le problème au problème de l'égalité des coefficients, au problème du zéro dans un coin et au problème de l'atteignabilité pour les fonctions affines par morceaux. Enfin, nous établissons des résultats de NP-complétude.

Mots-clés: Mortalité, Matrices, Décidabilité 


\title{
On the mortality problem for matrices of low dimensions
}

\author{
Olivier Bournez* and Michael Branicky**
}

${ }^{*} \mathrm{LIP}$

ENS-Lyon

46, Allée d'Italie

69364 LYON Cedex 07

FRANCE

obournez@ens-lyon.fr

\author{
**Electrical Engineering \\ and Applied Physics Department \\ Case Western Reserve University \\ Cleveland, OH 44106-7221 \\ USA \\ branicky@alum.mit .edu
}

\begin{abstract}
In this paper, we discuss the existence of an algorithm to decide if a given set of $2 \times 2$ matrices is mortal: a set $F=\left\{A_{1}, \ldots, A_{m}\right\}$ of $2 \times 2$ matrices is said to be mortal if there exist an integer $k \geq 1$ and some integers $i_{1}, i_{2}, \ldots, i_{k} \in$ $\{1, \ldots, m\}$ with $A_{i_{1}} A_{i_{2}} \cdots A_{i_{k}}=0$. We survey this problem and propose some new extensions: we prove the problem to be BSS-undecidable for real matrices and Turing-decidable for two rational matrices. We relate the problem for rational matrices to the entry equivalence problem, to the zero in the left upper corner problem and to the reachability problem for piecewise affine functions. Finally, we state some NP-completeness results.
\end{abstract}

\section{Introduction}

Several undecidability results are known about problems involving matrices [5, 13]. For example, given a finite set $F$ of matrices with integer entries, it is undecidable whether the semi-group generated by $M$ contains a matrix having a zero in the right upper corner [16], is free [10, 7], or contains the zero matrix [19]. These problems have been proved to be undecidable when restricted to $3 \times 3$ matrices. But for both of them the question of their decidability or undecidability when restricted to $2 \times 2$ matrices remains open [5].

In this paper, we focus on the decidability of the last problem. A set $F=$ $\left\{A_{1}, \ldots, A_{m}\right\}$ of $d \times d$ matrices is said to be mortal if there exist an integer $k \geq 1$ and some integers $i_{1}, i_{2}, \ldots, i_{k} \in\{1, \ldots, m\}$ with $A_{i_{1}} A_{i_{2}} \cdots A_{i_{k}}=0$. Hence, we focus on the following problem:

Open problem 1 Is the decision problem Mort $\mathbb{Q}_{\mathbb{Q}}(2)$ decidable?

- Instance: a finite set $F$ of $2 \times 2$ matrices with rational entries.

- Question: is F mortal?

The decidability of problem $\operatorname{Mort}_{\mathbb{Q}}(2)$ remains unknown despite a lot of interest (see $[14,15]$ for some references and discussions).

The question of the decidability of Mort $_{\mathbb{Q}}(2)$ was first mentioned as an open problem in [21] and was formulated as follows "Find an algorithm, which given a finite set $H$ of non-singular linear transformations of the complex plane and lines $L$ 
and $M$ through the origin, determines whether some product from $H$ maps $L$ onto M".

When $K \in\{\mathbb{R}, \mathbb{Q}\}$, the problem $\operatorname{Mort}_{K}(d)$ (respectively $\operatorname{Mort}_{K}(d, m)$ ) will denote the following decision problem:

- Instance: a finite set $F$ of $d \times d$ matrices with entries in $K$ (respectively a finite set $F$ of $m d \times d$ matrices with entries in $K$ ).

- Question: is $F$ mortal?

One of our motivation is the following: deciding the mortality problem is equivalent to deciding the controllability of switched linear systems: given a system of the form $x(t+1)=A(t, u) x(t)$, where for all $t$ the set of possible values of $A(t, u)$ is a finite set $F$ of $d \times d$ matrices, the question of mortality of $F$ corresponds to the controllability (to the origin) of such a system. Cf. [3].

One other motivation is to understand the frontier between decidability and decidability for discrete time dynamical systems: proving that $\operatorname{Mort}_{\mathbb{Q}}(2)$ is decidable or undecidable would really clarify the situation for discrete time hybrid and dynamical systems: $\mathrm{cf}[11]$ and [8]. For example the reachability problem for piecewise affine dynamical systems has been proved undecidable for 2-dimensional systems, but is open and related to the mortality problem (see section 4.3) for 1-dimensional systems [11].

Note that, if Mort $_{\mathbb{Q}}(2)$ turned out to be undecidable, it would surely give a way, which would extend the results of $[1,11,18,23]$, to simulate a Turing machine by a dynamical system of low dimension since most of the undecidability results known up to this date rely on simulations of Turing machines.

\section{Links between dimension and number of matrices}

Paterson proved in [19] that the problem restricted to $3 \times 3$ matrices is not decidable:

Theorem 1 ([19]) Mort $\operatorname{QQ}_{\mathbb{Q}}(3)$ is recursively unsolvable.

More precisely, Paterson proved in [19] that, if the Post Correspondence Problem (PCP) is undecidable with $p$ rules, then $\operatorname{Mort}_{\mathbb{Q}}(3,2 p+2)$ is undecidable.

Using the Modified Post Correspondence Problem (MPCP) instead of PCP, we improve this result and obtain:

Proposition 1 Suppose that the Post Correspondence Problem is undecidable with $p$ rules. Then problem Mort $_{\mathbb{Q}}(3, p+2)$ is undecidable.

Proof: The Post Correspondence Problem (PCP) is the following: "given a a finite set of couples of words $\left\{\left\langle U_{i}, V_{i}\right\rangle \mid i=1 \ldots p\right\}$, determine if there exist a sequence of indexes $i_{1}, i_{2}, \ldots, i_{k}$ in $\{1,2, \ldots, p\}$ with $U_{i_{1}} U_{i_{2}} \ldots U_{i_{k}}=V_{i_{1}} V_{i_{2}} \ldots V_{i_{k}}$ ". It is proved in [19] that, to any instance $\left\{\left\langle U_{i}, V_{i}\right\rangle \mid i=1 \ldots p\right\}$ of PCP can be associated a finite set $F=\left\{S, T, W\left(U_{j}, V_{j}\right), W^{\prime}\left(U_{j}, V_{j}\right)\right.$ for $\left.j=1, \ldots, n\right\}$ of rational matrices which satisfy:

1. $F$ is mortal if and only if there exists $i_{1}, i_{2}, \ldots, i_{k}$ in $\{1,2, \ldots, p\}$ with

$$
S W^{\prime}\left(U_{i_{1}}, V_{i_{1}}\right) W\left(U_{i_{2}}, V_{i_{2}}\right) \ldots W\left(U_{i_{k}}, V_{i_{k}}\right) T=0
$$

2. for all integers $i_{1}, i_{2}, \ldots, i_{k}$ in $\{1,2, \ldots, p\}$,

$$
S W^{\prime}\left(U_{i_{1}}, V_{i_{1}}\right) W\left(U_{i_{2}}, V_{i_{2}}\right) \ldots W\left(U_{i_{k}}, V_{i_{k}}\right) T=0
$$

if and only if $U_{i_{1}} U_{i_{2}} \ldots U_{i_{k}}=V_{i_{1}} V_{i_{2}} \ldots V_{i_{k}}$. 
We replace the Post Correspondence Problem (PCP) by the Modified Post Correspondence Problem (MPCP) ${ }^{1}$ [9]: "Given a finite set of couples of words $\left\{\left\langle U_{i}, V_{i}\right\rangle \mid i=1 \ldots p\right\}$, determine if there exists a sequence of indexes $i_{2}, \ldots, i_{k}$ in $\{1,2, \ldots, p\}$ with $U_{1} U_{i_{2}} \ldots U_{i_{k}}=V_{1} V_{i_{1}} \ldots V_{i_{k}}$ ". Since PCP can be solved by $p$ calls to MPCP, MPCP is undecidable with $p$ rules when PCP is.

There remains only to prove that MPCP with $p$ rules reduces to $\operatorname{Mort}_{\mathbb{Q}}(3, p+2)$. Observing that in MPCP the first index $i_{1}$ must be 1 , the set of matrices $F=$ $\left\{T, S W_{U_{1}, V_{1}}^{\prime}, W_{U_{j}, V_{j}}\right.$ for $\left.j=1, \ldots, n\right\}$ is mortal if and only if there exist $i_{2}, \ldots, i_{k}$ in $\{1,2, \ldots, p\}$ with $S W^{\prime}\left(U_{1}, V_{1}\right) W\left(U_{i_{2}}, V_{i_{2}}\right) \ldots W\left(U_{i_{k}}, V_{i_{k}}\right) T=0$, which in turns holds if and only if $\left\{\left\langle U_{i}, V_{i}\right\rangle \mid i=1 \ldots p\right\}$ is a positive instance of MPCP.

Now, the following result is proved in [2] and in [5]:

Lemma 1 [2, 5] For all $n \geq 2, m \geq 1, \operatorname{Mort}_{\mathbb{Q}}(d, m)$ undecidable implies $\operatorname{Mort}_{\mathbb{Q}}(d m, 2)$ undecidable.

The minimal number $p$ of rules for which PCP is undecidable is not known, but $p$ is an integer between 3 and 7 : see [17].

We obtain:

Corollary 1 The following problems are undecidable:

- $\operatorname{Mort}_{\mathbb{Q}}(3,9)$

- $\operatorname{Mort}_{\mathbb{Q}}(27,2)$

\section{On the decidability of $\operatorname{Mort}(2,2)$.}

We come back now to the decidability of the mortality problem for 2-dimensional matrices. We prove first that $\operatorname{Mort}_{\mathbb{R}}(2,2)$ is BSS-undecidable. Then we prove that $\operatorname{Mort}_{\mathbb{Q}}(2,2)$ is Turing-decidable.

We will use several times the following lemma:

Lemma $2 A$ finite set $F=\left\{A_{1}, \ldots, A_{m}\right\}$ of $2 \times 2$ matrices is mortal if and only if there exist an integer $k$ and integers $i_{1}, \ldots, i_{k} \in\{1, \ldots, m\}$ with $A_{i_{1}} \ldots A_{i_{k}}=0$, and

1. $\operatorname{rank}\left(A_{i_{j}}\right)=2$ for $1<j<k$,

2. $\operatorname{rank}\left(A_{i_{j}}\right)<2$ for $j \in\{1, k\}$.

Proof: Only the direct sense requires a proof. Suppose $F$ is mortal. There exists a null product $A_{i_{1}} \ldots A_{i_{k}}=0$ with $k$ minimal. We may assume $k \geq 2$, since otherwise the assertion is immediate. The matrices $A_{i_{1}}$ and $A_{i_{k}}$ of this product are non-singular because otherwise a null-product with few matrices could be obtained by multiplying $A_{i_{1}} \ldots A_{i_{k}}$ by their inverse(s).

Let $j \geq 1$ be the smallest integer with $\operatorname{rank}\left(A_{i_{j}}\right)<2$. From $A_{i_{1}} \ldots A_{i_{k}}=0$, it follows that matrix $A_{i_{1}} \ldots A_{i_{j}-1}$ sends the image $I$ of matrix $A_{i_{j}} \ldots A_{i_{k}}$ to 0 . Now, $I$ is also equal the image of $A_{i_{j}}$ and is of dimension 1. Indeed, firstly, $I$ is clearly included in the image of $A_{i_{j}}$, secondly, by definition of $k, I$ can not be of dimension 0 , and thirdly, the dimension of the image of $A_{i_{j}}$ is at most 1 because $\operatorname{rank}\left(A_{i_{j}}\right)<2$. Hence $A_{i_{1}} \ldots A_{i_{j}-1} A_{i_{j}}=0$, which implies $j=k$, which in turns implies the direct sense of the lemma.

\footnotetext{
${ }^{1}$ The difference between PCP and MPCP is that in the latter the first index $i_{1}$ must be equal to 1
} 


\subsection{BSS-undecidability of $\operatorname{Mort}_{\mathbb{R}}(2,2)$}

Talking about the decidability or undecidability of $\operatorname{Mort}_{\mathbb{R}}(2)$ requires to talk about machines that manipulate real-numbers.

One first approach would be consider the problem as a recursive analysis problem. However, this approach is non-appropriate because one can not decide whether a real number is equal to zero in this model [24].

A more natural approach is to consider the problem as a decidability problem for the real Turing machine proposed by Blum Shub and Smale in [4]. We assume that our reader knows this model: see [4] otherwise. Roughly speaking, a BSSmachine is a Random Access Machine [9] extended with real numbers: it has an unbounded number of real registers $x_{1}, \ldots, x_{n}, \ldots$ which can contain any real number in unbounded precision and a finite number of built-in constants $\lambda_{1}, \ldots, \lambda_{m}$. Its program is made of arithmetic operations between its real registers of type $x_{i}:=x_{j} \# x_{k}$, for $\# \in\{+,-, *, /\}$, or of type $x_{i}:=\lambda_{j}$, or of tests of type $x_{i} \# x_{j}$ with $\# \in\{>, \geq,<, \leq,=, \neq\}$. Let $\mathbb{R}^{\infty}=\cup_{i \in \mathbb{N}} \mathbb{R}^{i}$. An input $x \in \mathbb{R}^{\infty}$ (i.e. of type $x=\left(x_{1}, \ldots, x_{i}, 0, \ldots, 0, \ldots\right)$ for some $\left.i\right)$ is said to be accepted by the machine if the program of the machine eventually halts when started with its real registers set to $x$. A language $L \subset \mathbb{R}^{\infty}$ is said to be BSS-recursively enumerable if it consists of the accepted inputs of some BSS-machine. The language $L$ is said to be BSS-recursive if, in addition, its complement is BSS-recursively enumerable.

In other words, BSS recursive sets are those that can be decided using only arithmetical operations and tests. The reader should refer to [4] for more formal descriptions.

We recall a lemma proved in [4]: a set $S \subset \mathbb{R}^{n}$ for some $n$ is said to be a basic semi-algebraic set if there exists a finite number of polynomials of $n$ variables $p_{1}, p_{2}, \ldots, p_{n_{1}}, p_{1}^{\prime}, \ldots, p_{n_{2}}^{\prime}$ with $S=\left\{\left(x_{1}, \ldots, x_{n}\right) \mid p_{1}\left(x_{1}, \ldots, x_{n}\right)>\right.$ $\left.0 \wedge \ldots \wedge p_{n_{1}}\left(x_{1}, \ldots, x_{n}\right)>0 \wedge p_{1}^{\prime}\left(x_{1}, \ldots, x_{n}\right)=0 \wedge \ldots \wedge p_{n_{2}}^{\prime}\left(x_{1}, \ldots, x_{n}\right)=0\right\}$. A semi-algebraic set is a finite union of basic semi-algebraic sets.

Lemma 3 Let $L \subset \mathbb{R}^{\infty}$ be a BSS-recursively enumerable set. Then $L$ is a denumerable union of semi-algebraic sets.

Scketch of proof: Write $L=\cup_{t \in \mathbb{N}} A c c_{t}$, where $A c c_{t}$ is the subset of the inputs that are accepted by the machine at time $t$. Check that each subset $A c c_{t}$ is a semi-algebraic set: see [4] for the formal details.

The remaining arguments of this subsection are inspired from [12] (observe that their seems to be a close relation between mortality and stability [12]).

We start by the following preliminary result:

Lemma 4 Let $a, b, \lambda \in \mathbb{R}$ be some real numbers with $a^{2}+b^{2} \neq 0, \lambda \neq 0$. Let $\theta$ be an argument of complex number $a+i b$. The couple of matrices $F(a, b, \lambda)=\left\{A_{1}, A_{2}\right\}$ with

$$
A_{1}=\left(\begin{array}{ll}
a & -b \\
b & a
\end{array}\right) \quad A_{2}=\left(\begin{array}{ll}
-\lambda & 1 \\
0 & 0
\end{array}\right)
$$

is mortal if and only if there exists an integer $n \in \mathbb{N}$ with $\lambda=\tan (n \theta)$.

Proof: By lemma 2, $F(a, b, \lambda)$ is mortal if and only if there exists an integer $n \in \mathbb{N}$ with $A_{2} A_{1}^{n} A_{2}=0$. This is in true if and only if there exists a $n$th power of $A_{1}$ which sends the image of $A_{2}$ to its kernel. Since $\operatorname{Im}\left(A_{2}\right)=<(1,0)>$, $\operatorname{Ker}\left(A_{2}\right)=<(1, \lambda)>$, and since $A_{1}$ is the composition of an homothety and a rotation of angle $\theta$, this is true if and only if there exists an integer $n \in \mathbb{N}$ with $\lambda=\tan (n \theta)$.

The following observations are easy: 
Lemma 5 Let $\theta$ be a real number. Let $E(\theta)$ be the subset of $\mathbb{R}$ defined by

$$
E(\theta)=\{\lambda \mid \text { there exists an integer } n \in \mathbb{N} \text { with } \lambda=\tan (n \theta)\}
$$

1. $E(\theta)$ is a dense subset of $\mathbb{R}$ if and only if $\theta / \pi \notin \mathbb{Q}$.

2. There exist two rational number $a, b \in \mathbb{Q}$ such that any argument $\theta$ of complex number $a+i b$ satisfies $\theta / \pi \notin \mathbb{Q}$ : take for example $a=1$ and $b=2$ (see lemma $6)$.

3. When $\theta / \pi \notin \mathbb{Q}$, the complement $E^{c}(\theta)$ of $E(\theta)$ in $\mathbb{R}$ has an uncountable number of connected components: actually, every point of $E^{c}(\theta)$ is its own connected component.

We can now prove that $\operatorname{Mort}_{\mathbb{R}}(2,2)$ is BSS-undecidable:

Theorem 2 Mort $_{\mathbb{R}}(2,2)$ is BSS-recursively enumerable but is not BSS-recursive.

Proof: Since building a BSS-machine that semi-recognizes $\operatorname{Mort}_{\mathbb{R}}(2,2)$ is easy, the problem is BSS-recursively enumerable.

Representing the matrices by their coefficients, the space of the instances of problem $\operatorname{Mort}_{\mathbb{R}}(2,2)$ is $\mathbb{R}^{8}$. Denote by Pos $\subset \mathbb{R}^{8}$ (resp. by $N e g \subset \mathbb{R}^{8}$ ) the subset of the positive (resp. negative) instances of the problem. Using lemma 3 , we only need to prove that $N e g$ is not a countable union of semi-algebraic sets.

Let $a, b \in \mathbb{Q}$ with $a+i b=\rho e^{i \theta}, \theta / \pi \notin \mathbb{Q}$ like in lemma 5 . Let $\gamma: \mathbb{R} \rightarrow \mathbb{R}^{8}$ be the function that sends $\lambda \in \mathbb{R}$ to the couple of matrices $F(a, b, \lambda)$. By definition of $\gamma$, the image $I m_{\gamma}$ of $\gamma$ is an algebraic subset of $\mathbb{R}^{8}$ and $\gamma$ realizes an homeomorphism between $\mathbb{R}$ and $I m_{\gamma}$. By lemma $4, \gamma^{-1}(P o s)=E(\theta)$ and $\gamma^{-1}(N e g)=E^{c}(\theta)$. Since $\gamma$ is an homeomorphism, $E^{c}(\theta)$ and $\gamma\left(E^{c}(\theta)\right)=N e g \cap I_{\gamma}$ must have the same number of connected components: i.e., by remark 3 of lemma 5 , they must have an uncountable number of connected components.

Suppose $N e g=\cup_{i \in \mathbb{N}} S_{i}$ where each $S_{i}$ is a semi-algebraic subset. It follows $N e g \cap I m_{\gamma}=\cup_{i \in \mathbb{N}}\left(\operatorname{Im}_{\gamma} \cap S_{i}\right)$. Each of the $\left(\operatorname{Im}_{\gamma} \cap S_{i}\right)$ must be a semi-algebraic subset because it is the intersection between an algebraic set and a semi-algebraic set. Since a semi-algebraic set has a finite number of connected components, $N e g \cap I m_{\gamma}$ must have a countable number of connected components. This is impossible by the previous paragraph.

We get immediately:

Corollary 2 - For $n \geq 2, m \geq 2$, the problem Mort $_{\mathbb{R}}(n, m)$ is BSS-recursively enumerable but not BSS-recursive.

- $\operatorname{Mort}_{\mathbb{R}}(2)$ is BSS-recursively enumerable but not BSS-recursive.

However, observe that it is easy to extract the following fact from the proofs of the next section:

Theorem 3 Problem $\operatorname{Mort}_{\mathbb{R}}(2,2)$ restricted to matrices with real eigen-values is BSS-recursive.

Let us discuss the result of theorem 2 and of corollary 2: deciding whether a set of matrices is mortal using only arithmetical operations is not possible. But it does not mean that the problem can not be decided by an algorithm which uses non-arithmetical operations or which uses arguments about the semi-ring $K$ of the entries for $K \neq \mathbb{R}$.

Thus, using some number-theoretical arguments, we prove in the next subsection that the problem $\operatorname{Mort}_{\mathbb{Q}}(2,2)$ is Turing-decidable (that is, decidable in the classical sense). 


\subsection{Turing-decidability of $\operatorname{Mort}_{\mathbb{Q}}(2,2)$}

The decidability of $\operatorname{Mort}_{\mathbb{Q}}(2,2)$ has already be claimed [5, 20]. But the proofs was either wrong or incomplete (for example, contradicting the previous section) [20] or missing [5]. We present here a full proof.

The previous section shows that $\operatorname{Mort}_{\mathbb{Q}}(2,2)$ require the use of some numbertheoretical arguments. Actually, the arguments we will use are given by the following result extracted from [22]:

Lemma 6 ([22]) - The following decision problem is decidable:

- Instance:

* a rational number $p \in[-1,1]$

* a rational number $q \in[-1,1]$

- Question: does there exist $\theta \in \mathbb{R}$ and an integer $n \in \mathbb{N}$ with $\cos (\theta)=p$ and $\cos (n \theta)=q$ ?

- When $p \notin\{0,1 / 2\}$, there are at most a finite number of such $n$ and those $n$ can be computed effectively.

Proof: Write $p=r / s, q=u / v$ where $r, s, u, v$ are some integers with $g c d(r, s)=$ $\operatorname{gcd}(u, v)=1$. The decidability of the problem when $p=0$ or $p=1 / 2$ is trivial. Suppose $p \notin\{0,1 / 2\}$. $\operatorname{Cos}(n \theta)$ is a polynomial in $\cos (\theta)$ with integer coefficients. If this polynomial is written $\cos (n \theta)=p_{n}(r / s)$, then $s^{n} p_{n}(r / s)$ is some integer $c_{n}$ which satisfies

$$
2 r c_{n+1}-s^{2} c_{n}=c_{n+2}
$$

with $c_{1}=r$ et $c_{2}=2 r^{2}-s^{2}$ (if we denote $a_{n}=\sin (n x)$ and $b_{n}=\cos (n x)$, this recurrence comes from $a_{n+1}=a_{1} b_{n}+b_{1} a_{n}, b_{n+1}=b_{1} b_{n}-a_{1} a_{n}$, etc).

Suppose that $s$ is not a power of 2 . Write $s=2^{a} b, s^{\prime}=2^{a^{\prime}} b^{\prime}$ with $b>1, b^{\prime} \geq 1$ odd. We are searching an integer $n$ such that $c_{n} /\left(2^{a n} b^{n}\right)=u /\left(2^{a^{\prime}} b^{\prime}\right)$. We claim $\operatorname{gcd}\left(c_{n}, b^{n}\right)=1$ for all $n \in \mathbb{N}$. Indeed, if some odd integer $d$ divides simultaneously $s$ et $c_{n}$, then, since $g c d(r, s)=1$, the assertion $d\left|c_{n-1}, d\right| c_{n-2}, \ldots, d \mid c_{2}$ implies $d \mid r^{2}$, which in turns implies $d=1$. As a consequence an integer $n$ candidate must satisfy $b_{1}=b^{n}$. There are at most a finite number of such $n$ and those $n$ are computable.

Suppose now that $s$ is a power of 2. Write $s=2^{k}, k>1$ (remember that we supposed $r / s \neq 1 / 2)$. Write every $c_{n}$ as $c_{n}=2^{\lambda_{n}} v_{n}$ where $v_{n}$ is an odd integer. Recurrence (1) becomes

$$
2^{\lambda_{n+1}+1} r v_{n+1}-2^{\lambda_{n}+2 k} v_{n}=2^{\lambda_{n+2}} v_{n+2}
$$

We prove first that there exists an integer $n$ with $\lambda_{n}+1<2 k+\lambda_{n-1}$ : if it was false, we would always have $\lambda_{n}+1 \geq 2 k+\lambda_{n-1}$, so that $\lambda_{n}+1 \geq 2(n-1) k+\lambda_{1}$ would hold for all $n$. Since $|\cos (n \theta)|<1$, we have $k n \geq \lambda_{n}$ which implies $k n \geq$ $2(n-1) k+\lambda_{1}-1$ for all $n \in \mathbb{N}$. Clearly this is impossible.

Let $n_{0}$ be the smallest integer such that $\lambda_{n_{0}+1}<2 k+\lambda_{n_{0}}$ : $n_{0}$ can be computed effectively by testing this condition for increasing $n$. We have $\lambda_{n_{0}+2}=\lambda_{n_{0}+1}+1$, $\lambda_{n_{0}+2}+1=\lambda_{n_{0}+1}+2<\lambda_{n_{0}+1}+2 k$, so that for all integer $h \geq 0, \lambda_{n_{0}+2+h}=$ $\lambda_{n_{0}+1+h}+1$ holds. Hence, for each positive integer $h$, we must have $\lambda_{n_{0}+h}=\lambda_{n_{0}}+h$.

Now return to the existence of an integer $n$ with $\cos (n \theta)=u / v$. For $\cos (\theta)$ having denominator $2^{k}, v$ must be a power of 2. Suppose $v=2^{m}$. It may happen that there exists a solution for $n \leq n_{0}$. For $n>n_{0}$, a solution $n=n_{0}+h$ must satisfy $\cos \left(\left(n_{0}+h\right) \theta\right)=v_{n_{0}+h} 2^{\lambda_{n_{0}}+h} / 2^{k\left(n_{0}+h\right)}=u / 2^{m}$, hence $k\left(n_{0}+h\right)-\lambda_{n_{0}}-h=m$, or $h=\left(m+\lambda_{n_{0}}-k n_{0}\right) /(k-1)$. That is, the only integer $n$ candidate exceeding $n_{0}$ is $n_{0}+\left(m+\lambda_{n_{0}}-k n_{0}\right) /(k-1)$. Hence, there at most $n_{0}+2$ integers $n$ candidate to satisfy $\cos (n \theta)=u / v$ and those candidates are computable.

We get: 
Theorem 4 Problem Mort $_{\mathbb{Q}}(2,2)$ is decidable.

Proof: Let $F=\left\{A_{1}, A_{2}\right\}$ be an instance of the problem. Suppose without loss of generality that the rank of $A_{2}$ is greater than the rank of $A_{1}$. If $A_{1}$ is of rank 2, then the two matrices are non-singular and $F$ is non mortal by lemma 2. If $A_{1}$ is of rank 0 then $F$ is mortal. If the two matrices have rank 1, by lemma 2, it suffices to test whether one of the products $A_{1}^{2}, A_{1} A_{2}, A_{2} A_{1}, A_{2}^{2}$ is null.

There remains only the case where $A_{2}$ is non singular and $A_{1}$ is of rank 1 . By lemma $2, F$ is mortal if and only if there exists an integer $n \in \mathbb{N}$ with

$$
A_{1} A_{2}^{n} A_{1}=0
$$

We want to check this relation algebraically using the Jordan forms of the matrices $A_{1}$ and $A_{2}$. Write

$$
\begin{gathered}
A_{1}=P_{1}^{-1} J_{1} P_{1}, \quad A_{2}=P_{2}^{-1} J_{2} P_{2} \\
J_{1}=\left(\begin{array}{cc}
\kappa & 0 \\
0 & 0
\end{array}\right),
\end{gathered}
$$

and

$$
J_{2}=\left(\begin{array}{ll}
\lambda & 0 \\
0 & \mu
\end{array}\right) \quad \text { or } \quad\left(\begin{array}{ll}
\lambda & 1 \\
0 & \lambda
\end{array}\right) .
$$

with $P_{1}$ and $P_{2}$ non-singular. Eigen value $\kappa$ is rational since $\kappa$ is equal to the trace of rational matrix $A_{1}$. Eigen values $\lambda$ and $\mu$ are the (possibly complex) roots of the characteristic polynomial of rational matrix $A_{2}$.

Equation 3 becomes

$$
P_{1}^{-1} J_{1} P_{1} P_{2}^{-1} J_{2}^{n} P_{2} P_{1}^{-1} J_{1} P_{1}=0
$$

or, since $P_{1}$ is non-singular,

$$
J_{1} P J_{2}^{n} P^{-1} J_{1}=0
$$

where

$$
P=P_{1} P_{2}^{-1}=\left(\begin{array}{cc}
p & q \\
r & s
\end{array}\right)
$$

Now, after substituting $P, P^{-1}$ and $J$, when $J_{2}$ is of the first form, the problem is equivalent to testing whether there exists an integer $n \in \mathbb{N}$ with $p s \lambda^{n}-q r \mu^{n}=0$, and when $J_{2}$ is of the second form, the problem is equivalent to testing whether there exists an integer $n \in \mathbb{N}$ with $(p s-q r) \lambda^{n}-r p n=0$.

Suppose $A_{2}$ of the second form. Eigen value $\lambda$ is rational because $\lambda$ is equal to half the trace of rational matrix $A_{2}$. It follows that the coefficients $\kappa, p, q, r, s$ are easily computable rational numbers. Testing whether there exists an integer $n$ with $(p s-q r) \lambda^{n}-r p n=0$ is easy: compute numerically an approximation up to $1 / 2$ of real roots of equation $(p s-q r) \lambda^{x}-r p x=0$. It gives at most two integers $n$ candidate to be solution and check if one of the candidates is solution.

Suppose $A_{2}$ of the first form. We want to test the existence of an integer $n$ with $p s \lambda^{n}-q r \mu^{n}=0$. Observe that $\lambda \neq 0, \mu \neq 0$ since $A_{2}$ is of rank 2. $\lambda, \mu$ and the coefficients $p, q, r, s$ can be complex numbers but are computable elements of $\mathbb{Q}(\lambda)$ : that is, they are of the form $a+\lambda b$ for some computable rational numbers $a, b \in \mathbb{Q}$. By computing in $\mathbb{Q}(\lambda)$, the cases $p s=0$ or $q r=0$ are trivial. Suppose now $p s \neq 0$ and $q r \neq 0$. The problem is equivalent to testing whether there exists an integer $n$ with $(\lambda / \mu)^{n}=(p q) /(r s)$. We must have $|\lambda / \mu|^{n}=|p q| /|r s|$. When $|\lambda / \mu| \neq 1, n$ must be equal to $|p q| /(|r s| \log |\lambda / \mu|)$ and it suffices to compute 
numerically an approximation up to $1 / 2$ of this real quantity to get at most two integers $n$ candidate to be solutions. When $|\lambda / \mu|=1$ and $\lambda$ et $\mu$ are real numbers, we have necessarily $\lambda=\mu$ or $\lambda=-\mu$. In both cases, by computing in $\mathbb{Q}(\lambda)$ the problem is trivial. When $|\lambda / \mu|=1$ and $|p q| /|r s| \neq 1$ the problem has no solution.

There remains only the case where $\lambda$ and $\mu$ are two conjugated complex roots and $(p q) /(r s)$ is a complex number of modulus 1 . In that case $\lambda$ is a complex number with rational real part because $\lambda$ is a root of the characteristic polynomial of matrix $A_{2}$ with rational coefficients. Therefore, complex numbers $\lambda / \mu$ and $(p q) /(r s)$ of type

$a+\lambda b$ with computable $a, b \in \mathbb{Q}$ must also have rational computable real parts. If $\theta$ denotes an argument of complex number $\lambda / \mu$ of modulus 1 , an integer $n$ solution must satisfy $\cos (n \theta)=r^{\prime}$ where $r^{\prime}$ is the real part of $(p q) /(r s)$. When the real part $p^{\prime}$ of $\lambda / \mu$ is equal to $1 / 2, n \mapsto(\lambda / \mu)^{n}$ is a periodic sequence of period 6 : it suffices to check $(\lambda / \mu)^{n}=(p q) /(r s)$ for $n=0,1, \ldots, 5$. Case $p^{\prime}=0$ can be dealt similarly. Now, when $p^{\prime} \notin\{0,1 / 2\}$, by lemma 6 there are at most a finite number of integers $n$ satisfying $\cos (n \theta)=r^{\prime}$ and that integers are computable. It suffices to check if equation $(\lambda / \mu)^{n}=(p q) /(r s)$ holds for those integers.

We have just proved that $\operatorname{Mort}_{\mathbb{Q}}(2,2)$ is Turing-decidable. We do not know whether $\operatorname{Mort}_{\mathbb{Q}}(2,3)$ is decidable. So our knowledge of the decidability of $\operatorname{Mort}_{\mathbb{Q}}(2)$ stops at the previous theorem. However our proof of the BSS-undecidability of the problem shows that the problem is more a number-theoretical problem than an simple computability problem.

In next section, we will show that $\operatorname{Mort}_{\mathbb{Q}}(2)$ can be related to other open problems of the literature.

\section{Relations to other problems of the literature}

In this section we prove that $\operatorname{Mort}_{\mathbb{Q}}(2)$ is is equivalent to the equality of entry problem studied by [14], to the zero in the corner problem studied by $[16,5]$ and can be linked to the problems studied by [11].

When $C$ is a matrix, $C_{i, j}$ will denote the $j^{t h}$ coefficient of the $i^{t h}$ line of $C$.

\subsection{Equality of entries}

Here is a variation of theorem 2 of [14] (unlike theorem 2 of [14], we do not suppose $F$ to be made only of non-singular matrices):

Lemma 7 Let $F$ be a finite set of $2 \times 2$ matrices with rational entries. There exists an integer $k$ and some integers $i_{1}, \ldots, i_{k}$ such that $A_{i_{1}} \ldots A_{i_{k}}$ is a matrix $C$ satisfying $C_{2,1}=C_{2,2}$ if and only if the finite set $F^{\prime}$ made of the matrices of $F$ and of the matrix

$$
H=\left(\begin{array}{cc}
0 & 1 \\
0 & -1
\end{array}\right)
$$

is mortal.

Proof: First observe that $H C H=0$ holds if and only if $C_{2,1}=C_{2,2}$. That proves the direct sense.

Conversely, by lemma 2 , if $F$ is mortal there exist $i_{1}, \ldots, i_{k}$ with $A_{i_{1}} \ldots A_{i_{k}}=0$, $A_{i_{j}} \neq H$ for $1<j<k$, and $\operatorname{rank}\left(A_{i_{j}}\right)<2$ for $j \in\{1, k\}$. If $A_{i_{1}}=A_{i_{k}}=H$ the remark of the previous paragraph implies that $C=A_{i_{2}} \ldots A_{i_{k-1}}$ satisfies $C_{2,1}=$ $C_{2,2}$. If $A_{i_{1}} \neq H$ and $A_{i_{k}} \neq H$ then $A_{i_{1}} \ldots A_{i_{k}}$ is a product of matrices of $F$ equal to the null-matrix and the null-matrix $O$ satisfies $O_{2,1}=O_{2,2}$. Now, for the remaining cases, observe that equation $H C=0$ (resp. $C H=0$ ) implies $C_{2,1}=C_{2,2}$.

We can now extend a result of [14]: 
Theorem 5 (Equality of entries) Problem Mort $_{\mathbb{Q}}(2)$ is equivalent to the following decision problem:

- Instance: a finite set $F=\left\{A_{1}, \ldots, A_{m}\right\}$ of $2 \times 2$ matrices with rational entries.

- Question: does there exist an integer $k$ and some integers $i_{1}, \ldots, i_{k}$ such that $A_{i_{1}} \ldots A_{i_{k}}$ is a matrix $C$ satisfying $C_{2,1}=C_{2,2}$ ?

and to the following decision problem:

- Instance: a finite set $F=\left\{A_{1}, \ldots, A_{m}\right\}$ of non-singular $2 \times 2$ matrices with rational entries.

- Question: does there exist an integer $k$ and some integers $i_{1}, \ldots, i_{k}$ such that $A_{i_{1}} \ldots A_{i_{k}}$ is a matrix $C$ satisfying $C_{2,1}=C_{2,2}$ ?

Proof: Clearly the second problem reduces to the first. The first problem reduces to the mortality problem for $2 \times 2$ matrices by lemma 7 and a reduction from the mortality problem for $2 \times 2$ matrices to the second problem is given in [14].

\subsection{Zero in the left upper corner}

It is known that the problem of deciding whether the semi-group generated by a finite set of $3 \times 3$ non-singular matrices contains an element with a zero in the right upper corner is undecidable $[5,16]$. However, the decidability of the problem for $2 \times 2$ matrices is left open [5].

Nevertheless, this problem can be related to the mortality problem by:

Theorem 6 (Zero in left upper corner) Problem Mort $\mathbb{Q}_{\mathbb{Q}}(2)$ is equivalent to the following decision problem:

- Instance: a finite set $F=\left\{A_{1}, \ldots, A_{m}\right\}$ of $2 \times 2$ matrices with rational entries.

- Question: does there exist an integer $k$ and some integers $i_{1}, \ldots, i_{k}$ such that $A_{i_{1}} \ldots A_{i_{k}}$ is a matrix $C$ satisfying $C_{1,1}=0$ ?

and to the following decision problem:

- Instance: a finite set $F=\left\{A_{1}, \ldots, A_{m}\right\}$ of non-singular $2 \times 2$ matrices with rational entries.

- Question: does there exist an integer $k$ and some integers $i_{1}, \ldots, i_{k}$ such that $A_{i_{1}} \ldots A_{i_{k}}$ is a matrix $C$ satisfying $C_{1,1}=0$ ?

Proof: Denote

$$
P=\left(\begin{array}{ll}
0 & 1 \\
1 & 1
\end{array}\right)
$$

Observing that, for all matrix $C$, matrix $C^{\prime}=P C P^{-1}$ satisfies $C_{1,1}^{\prime}=0$ if and only if $C_{2,1}=C_{2,2}$, the above problems are equivalent to the equivalent problems of theorem 5 by conjugations by matrix $P$. 


\subsection{Restriction to lower triangular matrices}

It was proposed in [14] to restrict the previous problems to lower triangular matrices. Indeed [19] also proves that the equality of entry problem is undecidable for lower triangular $3 \times 3$ matrices with rational entries.

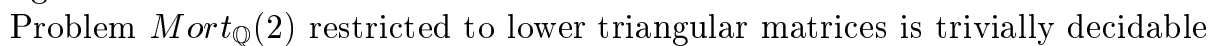
[14]: a finite set $F$ of lower triangular matrices is mortal if and only if there exist two matrices $A, B$ in $F$ with $A_{1,1}=0$ and $B_{2,2}=0$. The zero in the left upper corner problem when restricted to lower triangular matrices becomes also trivial.

However, the answer to the following question is not known:

Open problem 2 (Lower triangular matrices) Is the following decision problem decidable?

- Instance: a finite set $F=\left\{A_{1}, \ldots, A_{m}\right\}$ of non-singular lower-triangular $2 \times 2$ matrices with rational entries.

- Question: does there exist an integer $k$ and some integers $i_{1}, \ldots, i_{k}$ such that $A_{i_{1}} \ldots A_{i_{k}}$ is a matrix $C$ satisfying $C_{2,1}=C_{2,2}$ ?

We prove that this problem can be related to an non-deterministic version of the open problem mentioned in [11]:

Theorem 7 Open problem 2 is equivalent to the decidability of the following decision problem:

- Instance: a finite set $F=\left\{f_{1}, \ldots, f_{m}\right\}$ of non-constant rational affine functions of dimension 1 (i.e. a set of functions of type $f_{i}: x \mapsto a_{i} x+b_{i}, a_{i}, b_{i} \in \mathbb{Q}$, $\left.a_{i} \neq 0\right)$.

- Question: does there exists a composition $f_{i_{1}} \circ f_{i_{2}} \circ \ldots \circ f_{i_{k}}$ of these functions that maps point 0 to point 1 ?

Proof: Call this problem the composition problem. Suppose that a finite set $F=\left\{A_{1}, \ldots, A_{m}\right\}$ of non-singular lower-triangular matrices is given. Without loss of generality, we can suppose $A_{2,2}=1$ for each matrix $A \in F$. Indeed, each matrix $A \in F$ must satisfy $A_{2,2} \neq 0$ to be non-singular, and replacing each matrix $A$ by matrix $A / A_{2,2}$ in $F$ does not change the mortality of set $F$.

Problem 2 reduces to the instance $F^{\prime}=\left\{f_{1}, \ldots, f_{m}\right\}$ of the composition problem where $f_{i}: x \mapsto\left(A_{i}\right)_{1,1} x+\left(A_{i}\right)_{2,1}$ : any product $C=A_{i_{1}} \ldots A_{i_{k}}$ of lower-triangular matrices with $\left(A_{i_{j}}\right)_{2,2}=1$ satisfies $C_{2,2}=1$ and $C_{2,1}=f_{i_{1}} \circ f_{i_{2}} \ldots f_{i_{k}}(0)$.

Conversely the composition problem reduces to problem 2: when a finite set $F=\left\{f_{1}, \ldots, f_{m}\right\}$ of non-constant affine rational functions is given, $f_{i}: x \mapsto a_{i} x+b_{i}$, it suffices to consider $F^{\prime}=\left\{A_{1}, \ldots, A_{m}\right\}$ with

$$
A_{i}=\left(\begin{array}{cc}
a_{i} & 0 \\
b_{i} & 1
\end{array}\right)
$$

and to observe that any product $C=A_{i_{1}} \ldots A_{i_{k}}$ of matrices of this form satisfies $C_{2,2}=1$ and $C_{2,1}=f_{i_{1}} \circ f_{i_{2}} \ldots f_{i_{k}}(0)$.

\section{$5 \quad$ NP-completeness results}

\section{$5.1 \quad K$-length mortality}

A set $F=\left\{A_{1}, \ldots, A_{m}\right\}$ of $d \times d$ matrices is said to be $K$-length mortal if there exist an integer $k \leq K$ and some integers $i_{1}, i_{2}, \ldots, i_{k} \in\{1, \ldots, m\}$ with $A_{i_{1}} A_{i_{2}} \cdots A_{i_{k}}=$ 0 . 
Theorem 8 Given a set $F$ of $m 3 \times 3$ matrices with rational entries and an integer $K \leq 1+m / 2$ the decision problem 'Is $F K$-length-mortal?' is NP-hard.

Proof: Via the reduction of [19] (or the proof of proposition 1) and the NPcompleteness of Bounded PCP [6].

Observe that [2] proves that this result remains true whenever the matrices are assumed to have entries in $\{0,1\}$.

\subsection{Mortality without repetition}

When repetitions of matrices are not allowed, the problem becomes clearly also decidable: a set $F=\left\{A_{1}, \ldots, A_{m}\right\}$ of $d \times d$ matrices is said to be mortal without repetition if there exist integers $k \geq 1$ and some integers $i_{1}, i_{2}, \ldots, i_{k} \in\{1, \ldots, m\}$ such that $A_{i_{1}} A_{i_{2}} \cdots A_{i_{k}}=0$ and $i_{j_{1}} \neq i_{j_{2}}$ for all $j_{1} \neq j_{2}$.

Theorem 9 Given a finite set $F$ of $m 2 \times 2$ matrices, and an integer $K$, the decision problem "Is F K-length-mortal without repetition?" is NP-hard in the strong sense.

The proof uses a reduction from subset product [6]. We restate this problem here:

Proposition 2 (Subset Product (Yao)) Given a finite set $A$, a size s $(a) \in \mathbb{N}^{+}$ for each $a \in A$, and a positive integer $B$, the decision problem "Is there a subset $A^{\prime} \subset A$ such that the product of the sizes of the elements in $A^{\prime}$ is exactly $B$ ?" is NP-complete in the strong sense.

Proof:[of Theorem 9] Given an instance of subset product with $|A|=n$, define $n+3$ matrices as follows:

$$
\left(\begin{array}{cc}
1 & 0 \\
0 & s(a)
\end{array}\right), \text { for } a \in A, \quad\left(\begin{array}{cc}
1 & 0 \\
B & 1
\end{array}\right), \quad\left(\begin{array}{cc}
0 & 1 \\
0 & -1
\end{array}\right), \quad\left(\begin{array}{cc}
0 & 1 \\
0 & -1
\end{array}\right) .
$$

Note that we have repeated the last matrix, since we are required to use it twice. Denote the last matrix by $H$. Check that for all $2 \times 2$ matrix $A, H A H=0$ if and only if $A_{2,1}=A_{2,2}$. Hence, by lemma 2, this set of matrices is mortal without repetition with length $4 \leq k \leq n+3$ steps if and only if subset product has a solution in $1 \leq k-3 \leq n$ steps.

\section{Thanks}

We would like to thanks Eduardo Sontag for having pointed out the paper [12].

\section{References}

[1] E. Asarin, O. Maler, and A. Pnueli. Reachability analysis of dynamical systems having piecewise-constant derivatives. Theoretical Computer Science, 138(1):35-65, February 1995.

[2] V. D. Blondel and J. N. Tsitsiklis. When is a pair of matrices mortal? Information Processing Letters, 63(5):283-286, September 1997.

[3] V.D Blondel and J.N. Tsitsiklis. Complexity of stability and controllability of elementary hybrid systems. Automatica, 35(3), 1999.

[4] L. Blum, M. Shub, and S. Smale. On a theory of computation and complexity over the real numbers; $n p$ completeness, recursive functions and universal machines. Bulletin of the American Mathematical Society, 21(1):1-46, July 1989 . 
[5] J. Cassaigne and J. Karhumäki. Examples of undecidable problems for 2generator matrix semigroups. Theoretical Computer Science, 204(1):29-34, September 1998.

[6] M. R. Garey and D. S. Johnson. Computers and Intractability. W. H. Freeman and Co, 1979.

[7] T. Harju and J. Karhumaki. Morphisms. In Rozenberg, editor, Handbook of Formal Languages. Springer-Verlag, February 1997.

[8] T. A. Henzinger, P. W. Kopke, A. Puri, and P. Varaiya. What's decidable about hybrid automata? Journal of Computer and System Sciences, 57(1):94-124, August 1998.

[9] J. E. Hopcroft and J. D. Ullman. Introduction to Automata Theory Languages and Computation. Addison-Wesley, October 1979.

[10] D.A. Klarner, J.C. Birget, and W. Satterfield. On the undecidability of the freeness of integer matrix semigroups. Int. J. Algebra Comp., 1:223-226, 1991.

[11] P. Koiran, M. Cosnard, and M. Garzon. Computability with low-dimensional dynamical systems. Theoretical Computer Science, 132(1-2):113-128, September 1994 .

[12] V.S. Kozyakin. Algebraic unsolvability of problem of absolute stability of desynchronized systems. Scientific-Industrial Organization of Automatic Control Systems, 6:41-47, 1990.

[13] M. Krom. An unsolvable problem with products of matrices. Mathematical System Theory, 14:335-337, 1981.

[14] M. Krom and M. Krom. Recursive solvability of problems with matrices. Zwitschr. f. math. Logik und Grundlagen d. Math, 35:437-442, 1989.

[15] M. Krom and M. Krom. More on mortality. American Mathematical Monthly, 97:37-38, 1990.

[16] Z. Manna. Mathematical Theory of Computations. McGraw-Hill, USA, January 1974.

[17] Y. Matiyasevich and G. Sénizergues. Decision problems for semi-Thue systems with a few rules. In Proceedings, $11^{\text {th }}$ Annual IEEE Symposium on Logic in Computer Science, pages 523-531. IEEE Computer Society Press, 27-30 July 1996.

[18] C. Moore. Unpredictability and undecidability in dynamical systems. Physical Review Letters, 64(20):2354-2357, May 1990.

[19] M. S. Paterson. Unsolvability in 3x3 matrices. Studies in Applied Mathematics, XLIX(1):105-107, March 1970.

[20] Y. Saouter. The mortality of a pair of $2 \times 2$ matrices is decidable. Technical Report RR-2842, Institut National de Recherche en Informatique et en $\mathrm{Au}-$ tomatique, 1996.

[21] P. Schultz. Mortality of $2 \times 2$ matrices. American Mathematical Monthly, 84(2):463-464, 1977. Correction 85:p. 263,1978.

[22] H. S. Shank. The rational case of a matrix problem of harrison. Discrete Mathematics, 28:207-212, 1979. 
[23] H. T. Siegelmann and E. D. Sontag. On the computational power of neural nets. Journal of Computer and System Sciences, 50(1):132-150, February 1995.

[24] K. Weihrauch. A simple introduction to computable analysis. Technical Report 171-2/1995, Fernuniversität Gesamthochschule in Hagen, Fachbereich Informatik, Hagen, Allemagne, 1995. 